\title{
Analysis of dust losses in photovoltaic modules
}

\author{
J. Zorrilla-Casanova ${ }^{* 1}$, M. Piliougine ${ }^{1}$, J. Carretero ${ }^{1}$, P. Bernaola ${ }^{1}$, P. Carpena ${ }^{1}$, \\ L. Mora-López ${ }^{2}$, M. Sidrach-de-Cardona ${ }^{1}$ \\ ${ }^{1}$ Dpto. de Física Aplicada II, Universidad de Málaga, 29071 Málaga, Spain \\ ${ }^{2}$ Dpto. de Lenguajes y Ciencias de la Computación, Universidad of Málaga, 29071 Málaga, Spain \\ *Tel: 34- 95-213-2772 ,Fax: 34-95-213-1355, Email: ppz@ctima.uma.es
}

\begin{abstract}
The accumulation of dust on the surface of a photovoltaic module decreases the radiation reaching the solar cell and produces losses in the generated power. Dust not only reduces the radiation on the solar cell, but also changes the dependence on the angle of incidence of such radiation. This work presents the results of a study carried out at the University of Malaga to quantify losses caused by the accumulation of dust on the surface of photovoltaic modules. Our results show that the mean of the daily energy loss along a year caused by dust deposited on the surface of the PV module is around $4.4 \%$. In long periods without rain, daily energy losses can be higher than $20 \%$. In addition, the irradiance losses are not constant throughout the day and are strongly dependent on the sunlight incident angle and the ratio between diffuse and direct radiations. When studied as a function of solar time, the irradiance losses are symmetric with respect noon, where they reach the minimum value. We also propose a simple theoretical model that, taking into account the percentage of dirty surface and the diffuse/direct radiation ratio, accounts for the qualitative behavior of the irradiance losses during the day.
\end{abstract}

Keywords: optical losses, dust effects, energy losses

\section{Introduction}

The accumulation of dust on the surface of the photovoltaic modules decreases the incoming irradiance to the cell and produces power losses (see [1] and references therein). Previous studies [2] show that in dry areas, these losses could reach $15 \%$. In these cases the only solution is to clean the modules with water. In large-scale photovoltaic plants this task is often expensive, especially in those areas with water shortage.

Some approaches to analyze and quantify the effect of dust on photovoltaic modules have been proposed in the literature. The early studies about the relationship between dust and transmittance date back to a few decades ago, all of them in the context of solar thermal collectors. For example, in [3], the effect of dust on the irradiance received by various inclined surfaces of flat-plate collectors have been studied. The performances of one photovoltaic and two thermal panels during several months of outdoor exposure in Saudi Arabia have been measured in [4]. For the photovoltaic panel, the average degradation rate of the efficiency was 7\% per month. The authors of [5] made an experimental study of the effect of accumulation of dust on the surface of photovoltaic cells. Several kinds of dust having different physical properties were used. Experiments were performed using a solar simulator. They concluded that the results depend on many factors like the principal dust material, the size of dust particles and dust deposition density. We can see in [6] a co mputerized microscope system that has been developed for studying the physics of dust particles, which adhere to the surface of solar collectors and photovoltaic modules. The device enables investigators to calculate the particle size distribution of dust and the fraction of surface area covered by dust. Some examples are given for the use of such a measuring system for the study of photovoltaic and solar-thermal collector surfaces. Wind tunnel experiments were described in [7] to study the effect of wind velocity and air dust concentration on the drop of photovoltaic cell performance caused by dust accumulation on such cells. I-V characteristics were determined for various intensities of cell pollution. The evolutions of the $I_{s c}, V_{o c}, P_{\max }$, and $F F$ were examined. 
This work presents measurements of radiation losses produced by the accumulation of dust. The experiment has been carried out at the roof of the Photovoltaic Laboratory of the University of Málaga (latitude $36.7^{\circ} \mathrm{N}$, longitude $4.5^{\circ} \mathrm{W}$, altitude $50 \mathrm{~m}$ ) in the south of Spain. The campus is located between a residential and an industrial area surrounded by open fields with shrubs, weeds and some olive trees. Several roads with heavy traffic flow are very close to the building. At the time of the measurements, some excavations have been conducted in the vicinity of the building, which has increased the amount of inorganic dust particles present in atmospheric air.

\section{Methodology}

The objective of this work is to quantify losses caused by the accumulation of dust on the surface of photovoltaic modules. With this aim, irradiance values measured by two mSi cells have been recorded every ten minutes during a year. These cells have been previously calibrated against a reference pyranometer Kipp and Zonen CMP21. One of the reference cells has been cleaned daily, while the other has not been cleaned throughout the experiment (one year). Other parameters, such as rainfall and wind speed have been also measured.

Each reference cell has a low value shunt resistor between its terminals, and then the voltage drop across the shunt must be proportional to the short-circuit current and so it is further proportional to solar irradiance on the cell. The determination of the calibration constant for each cell is based on a comparison with a reference pyranometer under natural sun along a clear-sky day (only values of irradiance greater than $200 \mathrm{Wm}^{-2}$ have been taken into account). Both sensors (the reference cells and the pyranometer) are connected to an A/D module (cFP-AI-112) installed in Compact FieldPoint cFP-2120 data acquisition system that have been programmed to store a measure of all sensors at one-minute intervals. The manufacturer of the pyranometer provides a sensitivity constant that must be used to determine the actual irradiance value from the voltage its voltage output. Finally, a linear regression (setting offset to zero) between voltage values across each shunt and irradiance value have been performed to determine each constant.

Once the calibration procedure has been performed both cells remained installed and connected to the acquisition data system and measures of both of them have been recorded every three minutes along one year. The output value of each cell has been multiplied by the constant obtained by the calibration procedure in order to get the irradiance. Whereas one of them has been cleaned manually every day, the other cell has only been cleaned by rain. As well as registering irradiance values, the irradiation value along each day has been computed using trapezoidal integration too.

By comparing recorded irradiance values sensed by the two reference cells, dust influence on the received radiation can be quantified, and as consequence its effects on the solar energy received in the cell. Daily irradiation losses caused by dust are calculated comparing irradiation values sensed by the clean and the dirty cells. The two calibrated cells and the pyranometer are placed on a p lane whose tilt angle is $30^{\circ}$ (see Fig. 1). The period of measurements comprises from 12/15/08 to $12 / 14 / 09$. Along that period, summer was dry without any rain, and winter and spring had rainfall more frequent than usual for this period. An autumn with low rainfall completes the meteorological period. The availability of data for the studied period has been $96.4 \%$. 


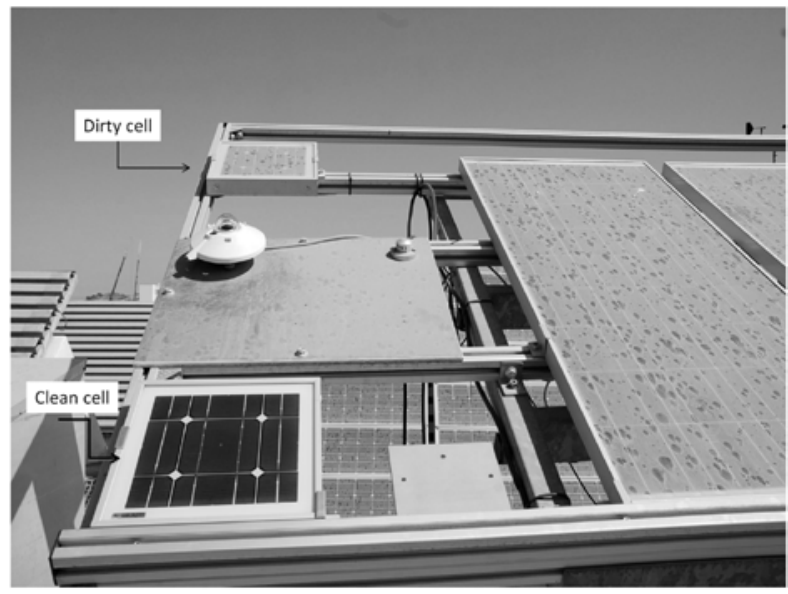

Fig. 1. The experimental setup used in the measurements.

\section{Results}

\subsection{Irradiation daily losses}

The evolution of the irradiation daily losses along the year of measurements is shown in Fig. 2. These losses $(H L)$ represent the fraction of daily energy that a PV module will not receive as consequence of dust deposited on their surface, and are calculated as

$H L(\%)=100 \times\left(\frac{H_{C C}-H_{D C}}{H_{C C}}\right)$

where $H_{C C}$ is the daily irradiation measured by the clean reference solar cell $\left(\mathrm{W} \mathrm{h} \mathrm{m}^{-2}\right)$ and $H_{D C}$ is the daily irradiation measured by the dirty cell $\left(\mathrm{W} \mathrm{h} \mathrm{m}^{-2}\right)$. As can be seen in Fig. 2 , the losses produced by the presence of dust are strongly dependent on the rainfall. In rainfall periods, a good cleaning of the dirty cell is produced and it recovers its initial performance; even a light rain, below $1 \mathrm{~mm}$, is enough to clean the cover glass, reducing daily losses $H L$ below $5 \%$. However, in long periods without rain, like summer, the accumulation of dust can cause daily losses exceeding $20 \%$.

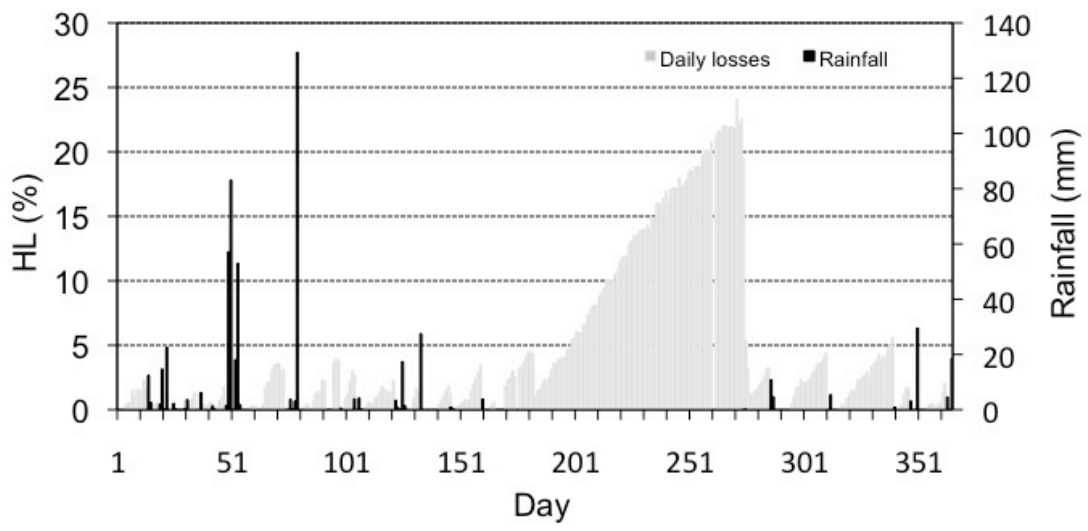

Fig. 2. HL values for all days of measurements along a whole year (left axis). We also plot daily values of rainfall (right axis).

The mean of the daily energy losses along a year caused by dust is $4.4 \%$. Monthly averages of daily energy losses are lower than $2 \%$ except in summer months, when the lack of rain favors the accumulation of dust and causes the increase of losses above $15 \%$. Note that the energy 
production reaches its maximum in these summer months, and therefore the possible adverse effect of dust is very relevant.

\subsection{Evolution of the dust losses along the day}

As seen in the last section, accumulations of dust on the surface of a photovoltaic module reduce strongly the energy received. We have not observed any influence of the wind speed or direction on the losses, probably because the high relative humidity contributes to the adherence of the dust particles on the module surface. As shown in previous studies [7-10], these losses should not be constant during the day, but have to be dependent on the incidence angle of beam radiation. In order to study this dependency, irradiation values sensed by the clean and the dirty cells throughout the day are compared. In this case, relative irradiance losses are calculated as:

$$
G L(\%)=100 \times\left(\frac{G_{C C}-G_{D C}}{G_{C C}}\right)
$$

where $G_{C C}$ is the irradiance value measured by the clean reference solar cell $\left(\mathrm{W} \mathrm{m}^{-2}\right)$ and $G_{D C}$ is the irradiance value measured by the dirty reference solar cell $\left(\mathrm{W} \mathrm{m}^{-2}\right)$. It should be pointed out that losses caused by the dependence of the transmission coefficient of the glass cover on the angle of incidence does not affect in the calculation of $G L$ since it is identical in both cells. However, the presence of dust modifies the angular dependence of the irradiance, which is different for the clean and the dirty cell, and precisely this effect is measured with GL.

These losses represent the fraction of irradiance that the cell will not receive, and in the case of PV modules, power losses. When cells are clean, losses are approximately constant during the day. As dirt is deposited on the dirty cell, the behavior of the losses is not constant throughout the day in clear sky days, becoming dependent on the angle of incidence. Daily evolution of dust losses on the 08/06/09 is shown in Fig. 3. This is a clear sky summer day, almost two months after the last rains; as consequence, dust level deposited on the dirty cell surface is high, causing daily losses of $14.8 \%$.

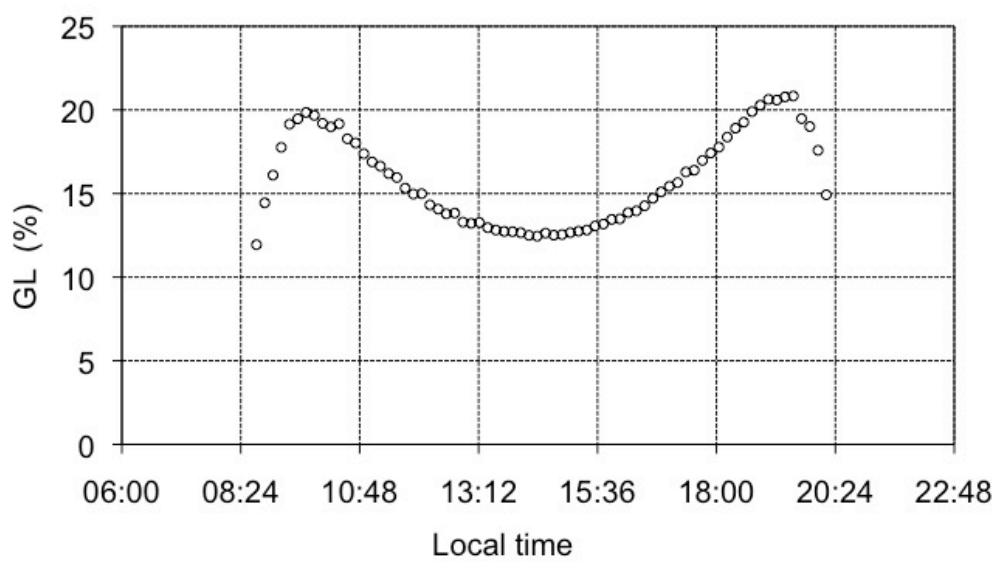

Fig. 3. Relative irradiance losses (GL) along a day.

The typical behavior of $G L$ as a function of the incident angle $(\theta)$ is shown in Fig. 4 . In this figure, we plot $G L$ curves obtained in several days with different $H L$ values (i.e. with different amounts of dust). As expected, losses are strongly dependent of the incident angle of radiation. Minimum transmittance losses occur at noon (12.4\%) when the incident angle is 
minimum. As incidence angle increases, losses increases slowly, but the growth rate increases as the angle. Nevertheless, from an angle of about $60^{\circ}, 1$ osses remain almost constant for a window of about $10^{\circ}$ and then, after a maximum of about $21 \%$, they decrease. This occurs at first and last hours of the day, when incidence angle is between $60-80^{\circ}$ and irradiance value is about $200 \mathrm{Wm}^{-2}$. (Note that morning maximum is slightly lower than afternoon maximum; the cause is that calibrated cells are no exactly in the same plane an there are a little bench between them). Dependence of dust losses with the angle is shown in Fig. 4.

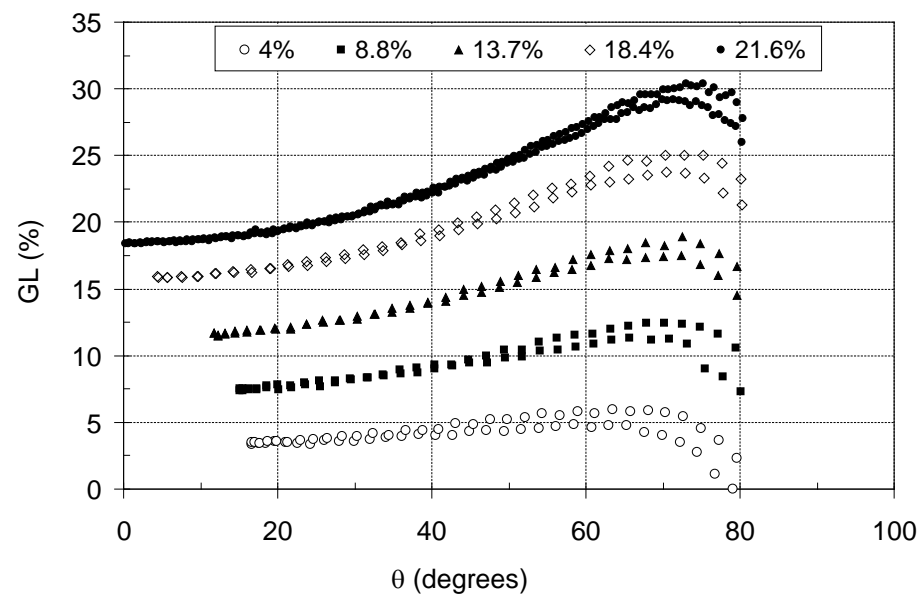

Fig. 4. Dependence of $G L$ with the angle of incidence $(\theta)$ for several days with different $H L$ values.

This behavior is related with the proportion of diffuse irradiance on global irradiance in the early morning and evening, when its value increases. In section 4, a theoretical model justifies this behavior. On cloudy days, when the global irradiance is mainly diffuse irradiance, losses remain almost constant throughout the day. Diffuse irradiance has not specific direction and hence losses are not dependent on the incidence angle.

Table I summarizes $H L, \theta$ and $G L$ at solar noon and the maximum value of $G L$ for each day shown in Fig. 4. Values of $H L$ are between $4.0 \%$ for the first day and $21.6 \%$ for the last day. It can be noticed that all the shape of the curves is generic and it is not dependent on the $H L$ value.

Table I. Measured parameters of Fig. 4

\begin{tabular}{ccccc}
\hline Date & $H L(\%)$ & $\begin{array}{c}\theta \text { at solar noon } \\
\text { (degrees) }\end{array}$ & $\begin{array}{c}G L \text { at solar } \\
\text { noon }(\%)\end{array}$ & $\begin{array}{c}G L \text { maximum } \\
(\%)\end{array}$ \\
\hline $27 / 06 / 2009$ & 4.0 & 17.0 & 3.5 & 5.9 \\
$13 / 07 / 2009$ & 8.8 & 15.1 & 7.5 & 12.5 \\
$30 / 07 / 2009$ & 13.7 & 11.7 & 11.7 & 18.9 \\
$23 / 08 / 2009$ & 18.4 & 4.4 & 16.0 & 25.0 \\
$04 / 09 / 2009$ & 21.6 & 0.2 & 18.4 & 30.4
\end{tabular}

\section{Modeling the losses produced by the dirt}

We have developed a simple model to justify the shape of the typical behaviour of the relative transmittance losses due to the presence of dust in the solar cell (see Fig. 3). The model is based on the following assumptions: 
a) Dust grains are modelled as spheres homogeneously distributed on the surface of the panel.

b) Each sphere has a reflection coefficient $R$, which accounts for both specular and diffused reflection.

c) Total incoming radiation from the Sun $\left(I_{T}\right)$ is composed of direct radiation $\left(I_{0}\right)$ and diffuse radiation $\left(I_{D}\right)$. We consider that this latter radiation comes homogeneously from any direction and it is kept constant along the day. Note that the total irradiance received by a clean solar cell is given by:

$$
G_{C C}=I_{0} \cos \theta+I_{D}
$$

where $\theta$ is the angle of incidence of direct radiation on the panel. The albedo radiation has been neglected.

d) In the dirty solar cell, any sphere of dust shadows the panel thus reducing the light reaching it. However not all radiation reaching the spheres is lost because part of if is reflected (a factor $R$ ) and can be partially recovered by the panel. Both effects, the shadowing and the recovery of light, depend on the angle of incidence of the direct radiation and thus vary along the day. On the other hand, there is no such dependence in the diffuse radiation since we assume that $I_{D}$ is constant along the day.

To quantify the irradiance losses GL due to the presence of dust in the solar cell we use Eq. (2). In order to understand the effect of the dust on the losses we have to analyse separately the direct and diffuse radiations.

\subsection{Direct radiation}

For the direct radiation the shadowing increases with the angle of incidence, reaching the maximum for $\theta=90^{\circ}$. At the same time, the fraction of the light specularly reflected reaching the panel increases with $\theta$ up to a maximum value and finally decreases for very large $\theta$. On the other hand, the fraction of light diffusely reflected reaching the panel is constant because the direction of the reflected rays is independent of the angle of incidence. The sum of all these contributions is not evident and therefore we simulate the phenomenon using a ray tracer [11]: For each angle of incidence $\theta$ we trace $10^{6}$ rays reaching a square cell of unit area with a single sphere on its center. We impose periodic boundary conditions. The reflection coefficient of the spheres is set to $R=65 \%(19.5 \%$ specular and $45.5 \%$ diffuse $)$, and the radius of the sphere is set to $r=0.315$ units, which is equivalent to a coverage of $31.17 \%$ of the surface of the cell. In Fig. 5 we show GL as a function of the angle of incidence for this simulation (dashed line). The values of the parameters are physically acceptable and have been chosen in order to fit the experimental results (also shown in Fig. 5) for small angles of incidence. As can be observed, the model does not reproduce at all the behaviour of GL for large angles of incidence. In particular it gives a monotonously increasing $G L$ while the experimental one reaches a maximum and decreases for very large angles of incidence.

\subsection{Diffuse radiation}

As we see, the contribution of direct radiation alone does not suffice to explain the experimental results. Therefore we incorporate the diffuse radiation to the model. Again we generate and trace $10^{6}$ rays with directions uniformly distributed. The total amount of energy carried by these rays is equivalent to $23 \%$ of the total radiation that would reach the cell under normal incidence. When these rays are included in the simulation (solid line in Fig. 5) 
the result obtained for GL agrees fairly well with the experimental results and in particular, it reproduces the reduction of the losses observed at large angles of incidence.

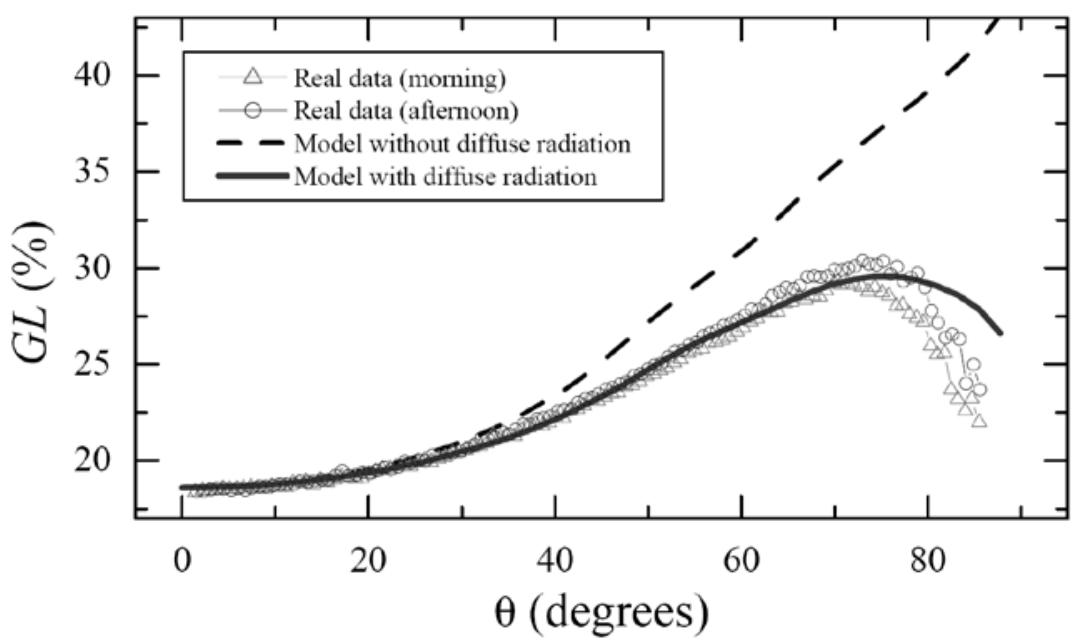

Fig.5. Relative irradiance losses of a dirty solar cell as a function of the angle of incidence of solar direct radiation. Hollow triangles (circles) correspond to real data measured in morning (afternoon) hours on the $4^{\text {th }}$ of September, 2009 (Malaga, Spain). Dashed line corresponds to the results obtained with a simulation (see text for details) in which diffuse radiation is not considered whereas the solid one has been obtained by taking into account this radiation.

\section{Conclusions}

In this work we have studied in general the energy losses due to accumulated dust on the surface of photovoltaic modules. First, we present results about daily irradiation losses and we show that the mean value of this quantity along a whole year is about $4.4 \%$. In rainfall periods, the rain water cleans the dirty cell and it recovers its normal performance: even a light rain, below $1 \mathrm{~mm}$, is enough to clean the cover glass, reducing daily losses $H L$ clearly below the average value of $4.4 \%$. However, in long periods without rain, like summer, the accumulation of dust can cause daily losses exceeding $20 \%$.

Second, we present results of the dust-caused irradiance losses $G L$ and its dependence on the angle of incidence $\theta$. The curve that describes the dependence of these losses on the angle of incidence has a very specific shape: $G L$ has a minimum at solar noon, then increases with $\theta$ up to a maximum value found when $\theta \approx 75^{\circ}$, and then decreases for larger values of $\theta$. This behavior can be explained by the influence of the diffuse radiation.

In addition, we have presented a simple model, simulated with the ray-tracing technique, to explain the behavior of losses in solar modules due to the presence of dust. With this model we have shown the relevance of diffuse radiation in order to understand the full behavior of losses as a function of the angle of incidence. Indeed, when only direct radiation is considered the model does not provide results comparable to experimental measures. On the other hand, when diffuse radiation is also taken into account, the model reproduces quite well the shape of the experimental data for reasonable values of the input parameters.

We conclude that the estimation of energy losses produced by the presence of dust have to be calculated in a different way for photovoltaic systems with fixed modules or with solartracking. In addition, the proportion of the diffuse component in the global radiation must be taken into account when estimating the energy losses produced by the dust on the system energy performance. 
Finally, it is very important to quantify energy losses produced by dust in dry areas where such losses could reach large values and so producing a substantial decrease in the efficiency of photovoltaic systems. In these cases a regular cleaning of the modules would be necessary thus increasing maintenance costs.

\section{Acknowledgements}

We acknowledge the Spanish "Ministerio de Ciencia e Innovación" (grant No. ENE07-67248) and "Junta de Andalucía" (grant No. P07-RNM-02504) for financial support.

\section{References}

[1] M. Mani, R. Pillai. Impact of dust on s olar photovoltaic (PV) performance: Research status, challenges and recommendations, Renewable and Sustainable Energy Reviews 14, 2010, pp. 3124-3131.

[2] M. Piliougine, J. Carretero, M. Sidrach-de-Cardona, D. Montiel, P. Sánchez-Friera. Comparative analysis of the dust losses in photovoltaic modules with different cover glasses. Proceedings of 23rd European Solar Energy Conference, 2008, pp. 2698-2700.

[3] H.P. Garg, Effect of dirt on transparent covers in flat-plate solar energy collectors, Solar Energy 15 (4), 1974, pp. 299-302.

[4] S.A.M. Said, Effects of dust accumulation on performances of thermal and photovoltaic flat-plate collectors, Applied Energy 37 (1), 1990, pp. 73-84.

[5] M.S. El-Shobokshy, F.M. Hussein, Effect of dust with different physical properties on the performance of photovoltaic cells, Solar Energy 51 (6), 1993, pp. 505-511.

[6] S. Biryukov, D. Faiman, A. Goldfeld, An optical system for the quantitative study of particulate contamination on solar collector surfaces, Solar Energy 66 (5), 1999, pp. 371-378.

[7] D. Goossens, E. Van Kerschaever, Aeolian dust deposition on phot ovoltaic solar cells: the effects of wind velocity and airborne dust concentration on cell performance, Solar Energy 66 (4), 1999, pp. 277-289.

[8] N. Martin, J.M. Ruiz, Calculation of the PV modules angular losses under field conditions by means of an analytical model, Solar Energy Materials \& Solar Cells 70, 2001, pp. 25-38.

[9] M. García, L. Marroyo, E. Lorenzo, M. P érez, Soiling and other optical losses in solar-tracking PV plants in Navarra, Progress in Photovoltaics: Research and Applications, DOI: 10.1002/pip.1004.

[10]N. Martin, J.M. Ruiz, Annual angular reflection losses in PV modules. Progress in Photovoltaics: Research and Applications 13, 2005, pp. 75-84.

[11] A.S. Glassner (Ed), An introduction to ray tracing, Academic Press, 1993. 